\title{
Managing Uncertainty in Macrocognition: A Multi-disciplinary Review and Integration
}

\author{
Michael A. Rosen \\ Department of Psychology, and the Institute for Simulation \\ and Training, University of Central Florida \\ mrosen@ist.ucf.edu
}

\author{
Stephen M. Fiore \\ Cognitive Sciences Program, and the Institute for \\ Simulation and Training, University of Central Florida \\ sfiore@ist.ucf.edu
}

\author{
Eduardo Salas \\ Department of Psychology, and the Institute for Simulation and Training, University of Central Florida \\ esalas@ist.ucf.edu
}

\begin{abstract}
Motivation. Uncertainty plays a major role in decision making and problem solving tasks in naturalistic environments and has received much interest across research disciplines. Research Approach. This poster provides a multi-disciplinary review of uncertainty. Findings/Design. Across disciplines, a view of uncertainty as an affective experience and as a resource that is managed and not always reduced has emerged. A framework rooted in group communication theory and emotion regulation is presented that outlines this process. Research limitations/Implications. Implications of this framework suggest that emotion regulation may be as important as the cognitive work performed by experts. However, these implications are in need of validation in applied contexts. Originality/Value. To date, there has been little cross-fertilization of ideas between the NDM and group communication communities of practice even though they investigate similar phenomenon. Take away message. Understanding complex phenomenon such as macrocognition in teams can be advanced by considering multi-disciplinary perspectives.
\end{abstract}

Keywords

Macrocognition in teams, uncertainty, team decision making

\section{INTRODUCTION}

Uncertainty is a central component of naturalistic models of decision making and a defining characteristic of NDM environments as well as emerging macrocognitive frameworks. In contrast to traditional decision making approaches that conceptualize uncertainty in terms of explicit probability assessments, uncertainty in NDM contexts has been defined as a 'sense of doubt that blocks or delays action' (Lipshitz \& Strauss, 1997, p. 150). In this way, uncertainty is viewed, at least in part, as an affective appraisal, as a feeling of uncertainty.

However, uncertainty in NDM is still largely viewed as a quantity to be reduced or eliminated, an obstacle to be overcome. While this is no doubt valid in many senses, it is a limited view consistent more with prescriptive decision making research traditions than with naturalistic ones. Uncertainty is inherent in macrocognitive task environments and can rarely be reduced in an absolute sense. Research from parallel traditions studying group and individual knowledge building and problem solving in naturalistic contexts have noted that reducing uncertainty is only one of several strategies adopted when managing complex tasks. A more comprehensive view considers uncertainty as a resource that individuals and teams actively manage through various cognitive and behavioral strategies. This includes reduction at times, and active cultivation at others. For example, uncertainty about the likelihood of highly negative events is frequently increased in group discussion. Similarly, an individual or group can actively increase uncertainty about a piece of information by questioning the credibility of the source of that information. This can occur when information does not fit a working understanding of the situation. Situations such as these are an integral part of macrocognition, but remain largely unaddressed by current models of how people cope with uncertainty.

\section{KEY ISSUES}

Therefore, this poster attempts to contribute to the understanding of uncertainty in real world situations through a critical review of the multi-disciplinary literature pertaining to the experience and management of uncertainty by teams and individuals in naturalistic settings. The long tradition of naturalistic research dealing with uncertainty in group communication theory will be synthesized with NDM approaches to understanding the role of uncertainty in complex cognitive work in individuals and teams. Specifically, this poster addresses the following goals. First, we describe 
various theoretical approaches to individual and team or group uncertainty management, uncertainty reduction, and problematic integration approaches. Second, we illustrate how the tools of multi-level theory construction and evaluation can help bring parsimonious order to the diverse literature relevant to this issue. Third, we advance a theoretical framework and set of propositions detailing how uncertainty management processes unfold within and between levels of analysis and ultimately influence outcomes. This is an input-process-output framework which includes inputs (e.g., goal conflict, motivation to reduce uncertainty) individual and team level behavioral and cognitive strategies (e.g., information seeking and avoiding behaviors), moderators (e.g., regulatory resources, trait affect), collaborative problem solving outcomes, and relations between these variables. This framework describes situations in which certain uncertainty management strategies will be more likely than others.

\section{CONCLUDING REMARKS}

In sum, this poster contributes a framework of uncertainty management rooted in the NDM tradition. This framework bridges disciplinary boundaries by drawing on group communication theory and explicitly articulates the role of affect management in cognitive work. In complex collaborative and macrocognitive environments, reducing uncertainty is only part of the story. Exclusive reduction of uncertainty can lead to an incomplete analysis of the problem space (e.g., the classic work on group think). Understanding how individuals and groups affectively experience and regulate uncertainty is a topic in need of further research.

\section{ACKNOWLEDGMENTS}

The views herein are those of the authors and do not necessarily reflect those of the organizations with which they are affiliated or their sponsoring agencies. This research was supported by the Office of Naval Research Collaboration and Knowledge Interoperability (CKI) Program and ONR MURI Grant \#N000140610446 (Dr Michael Letsky, Program Manager).

\section{REFERENCES}

Lipshitz, R., \& Strauss, O. (1997). Coping with uncertainty: A naturalistic decision-making analysis. Organizational Behavior and Human Decision Processes, 69(2), 149-163. 